\title{
(Tschechischer) Stolz und (bestätigte) Vorurteile. Eine Bilanz der tschechischen EU-Ratspräsidentschaft
}

\author{
Petr Kratochvíl und Vit Beně̌*
}

Jede EU-Ratspräsidentschaft hat ihren Teil an Krisen und unerwarteten Entwicklungen im Integrationsprozess zu bewältigen. Doch noch vor Beginn der tschechischen Ratspräsidentschaft sagten Kommentatoren voraus, dass die ersten sechs Monate des Jahres 2009 aus verschiedenen Gründen außerordentlich schwierig werden würden. Erstens würde die Europäische Union in einer ungeklärten institutionellen Situation (der noch zu ratifizierende Vertrag von Lissabon, die Wahlen zum Europäischen Parlament im Juni und die Vorbereitungen für die neue Europäische Kommission) weiterarbeiten. Zweitens gäbe es keinerlei Hoffnung, dass die wirtschaftliche Situation sich zum Besseren wenden würde oder dass die Mitgliedstaaten der Europäischen Union plötzlich zu mehr Einigkeit finden würden, wobei sie bislang überdeutlich nicht zu gemeinsamen Krisenbekämpfungsmaßnahmen in der Lage gewesen waren. Drittens wurde eine Reihe tief greifender Änderungen im Umfeld der Europäischen Union erwartet (eine neue US-Regierung, das immer , herrischere ' Russland), was die Notwendigkeit für eine starke EU-Führung noch betonte. Neben all diesen Faktoren hatte die tschechische Ratspräsidentschaft eine vierte Herausforderung zu bewältigen, nämlich die des stereotypen Bildes des Landes als eines eher kleinen, nach innen gerichteten, euroskeptischen, postkommunistischen osteuropäischen Staates ohne vorherige Erfahrung mit einer derart großen Aufgabe.

Doch was sich rückblickend als größter Stolperstein für die Ratspräsidentschaft herausstellte, war innenpolitischer Natur - das fehlende Verständnis tschechischer Politiker dafür, wie sehr innere Entwicklungen der Tschechischen Republik und die Europäische Union miteinander verknüpft sind. Das deutlichste Beispiel war die fehlende Vorausschau der Oppositionsführer dafür, welche enormen Auswirkungen ein erfolgreiches Misstrauensvotum während der Ratspräsidentschaft auf den Erfolg dieser Präsidentschaft haben würde. ${ }^{1}$ Unglücklicherweise war der Eifer der Regierung, ,Eindruck zu machen', genauso groß wie die Unfähigkeit der Oppositionsparteien, die Konsequenzen ihres Handelns umfassend zu begreifen. Die Regierung kümmerte sich nur wenig darum, wie ihre Sichtweisen auf europäischer oder internationaler Ebene rezipiert wurden. Paradoxerweise lösten diese Entwicklungen einen zweifachen Wechsel auf der innenpolitischen Ebene aus - erstens erlebte die bisher euroskeptische ODS (Demokratische Bürgerpartei, die größte rechts gerichtete Partei des Landes) einen Prozess einer eher unerwarteten Europäisierung. Zweitens wurden die radikalen tschechischen Euroskeptiker unter Führung von Staatspräsident Václav Klaus, der jegliche Vertiefung der Integration ablehnt, weiter marginalisiert.

Die folgende detaillierte Analyse der tschechischen Ratspräsidentschaft umfasst vier Teile. Die ersten beiden befassen sich mit der praktischen Arbeit der Ratspräsidentschaft, sowohl was die Handlungseffizienz (Teil eins) als auch die Verwirklichung der Prioritäten

* Dr. Petr Kratochvíl, Stellvertretender Direktor, Institute of International Relations, Prag.

Dr. Vít Beneš, Wissenschaftlicher Mitarbeiter, Institute of International Relations, Prag.

1 Ein weiteres Beispiel war der Gebrauch von zwei offiziellen Mottos der Ratspräsidentschaft. Die tschechische Diplomatie verwendete den Slogan „Europa ohne Grenzen“ gegenüber ihren Partnern, doch ein anderes, ironisches, schwer übersetzbares Wortspiel „Wir werden es süß machen für Europa“ richtete sich an die tschechische Gesellschaft. 
der Ratspräsidentschaft (Teil zwei) angeht. Unter Berücksichtigung der Tatsache, dass Erfolg oder Misserfolg einer Ratspräsidentschaft jedoch nicht ausschließlich von diesen beiden Kriterien abhängen, sondern eher von der allgemeinen Wahrnehmung der Ratspräsidentschaft durch die Schlüsselakteure (wie EU-Institutionen, EU-bezogene Think-Tanks, wichtige Medien und Mitgliedstaaten), wird ein Teil den externen Perzeptionen der Ratspräsidentschaft (Teil drei) gewidmet. Weil die Ratspräsidentschaft zweifelsohne lang andauernde Auswirkungen auf die Haltung der Tschechischen Republik gegenüber der Europäischen Union haben wird, wird zum Abschluss ein Sonderteil den innenpolitischen Auswirkungen der Ratspräsidentschaft gewidmet (Teil vier).

\section{Das Management der Ratspräsidentschaft: unsichtbar, aber verlässlich}

Die Vorbereitungen für die Ratspräsidentschaft begannen ziemlich früh, ungefähr zwei Jahre vor dem eigentlichen Turnus. So wurden anlässlich der Ratspräsidentschaft nach und nach einige institutionelle Änderungen vorgenommen, deren größte die der Schaffung der Position des Vizepremierministers für Europäische Angelegenheiten war. Dessen Büro wurde die zentrale Koordinationseinheit nicht nur während der Planungsphase, sondern auch während der Ratspräsidentschaft selbst. ${ }^{2}$ Diese Änderung hätte sicherlich erhebliche Positionskämpfe zwischen dem neuen Amt und dem Außenministerium, das den bedeutendsten Teil seiner Aufgaben verlor, ausgelöst. Doch weil der damalige Außenminister Karel Schwarzenberg und seine ihm vertrautesten Stellvertreter (wie Tomáš Pojar) enge Freunde des neu ernannten Vizepremierministers Alexandr Vondra waren, vollzog sich dieser Übergang überraschend geräuschlos.

Die Abteilungen des Außenministeriums, die sich mit der Europäischen Union befassten, wurden jedoch nicht aufgelöst, was zu zahlreichen Überschneidungen und Problemen wegen unklarer Verantwortlichkeiten führte. Ähnliche Veränderungen betrafen die anderen Ministerien ebenso wie die tschechische Ständige Vertretung in Brüssel: circa 340 neue Mitarbeiter wurden eingestellt, um während der Ratspräsidentschaft in den Ministerien zu arbeiten, und weitere 100 in der Ständigen Vertretung wie auch in den wichtigsten tschechischen Botschaften. Obwohl die Ständige Vertretung und vor allem ihre Leiterin Milena Vicenová wegen ihrer Unauffälligkeit kritisiert wurden, war die Staatsbürokratie verglichen mit Politikern und Diplomaten wahrscheinlich am besten vorbereitet und waren es die Beamten der mittleren Ebene, die in der Lage waren, das System sogar in Krisenzeiten am Laufen zu halten.

Dies war vor allem der Fall in der Zeit nach dem Sturz der Regierung. Nahezu alle geplanten Aktivitäten wurden realisiert und die Mitarbeiter des Verwaltungsapparates waren am wenigsten von den plötzlichen politischen Änderungen betroffen. Auch hinsichtlich der Kommunikation mit ihren Partnern in anderen Mitgliedstaaten waren die tschechischen Beamten ziemlich effektiv und versorgten ihre Partner schnell mit den benötigten Informationen. Dies gilt vor allem für die Mitarbeiter der Prager Ministerien (Außenministerium, Büro des Ministers für Europäische Angelegenheiten), weniger jedoch für diejenigen, die in Brüssel arbeiteten und oft mit der Kommunikation mit Prag überlastet und daher weniger in der Lage waren, EU-Stellen mit einem schnellen Feedback zu versorgen.

Die gleiche positive Beurteilung erstreckt sich auch auf die organisatorische Unterstützung der präsidentschaftsbezogenen Treffen, vor allem solche, die in der Tschechischen Republik stattfanden: eine Sitzung der tschechischen Regierung mit der Europäischen Kom-

2 Informationen über das Büro des Ministers für Europäische Angelegenheiten sind abrufbar unter: http:// www.vlada.cz/en/evropske-zalezitosti/european-affairs-17539/ (letzter Zugriff: 22.09.2009). 
mission, vier Gipfeltreffen (der EU-USA-Gipfel, der informelle Beschäftigungsgipfel, das Gipfeltreffen zur Östlichen Partnerschaft und der Gipfel zum ,Südlichen Korridor' für Energie und Verkehr), 14 informelle Treffen auf Ministerebene und zahllose andere Treffen auf niedrigerer Ebene. Wenngleich einige dieser Treffen politisch ziemlich sensibel waren (vor allem solche, an denen der tschechische Staatspräsident teilnahm), wurde deren Organisation von den Teilnehmern in der Regel als sehr gelungen bezeichnet. ${ }^{3}$ Ein Faktor, der geholfen haben mag, das positive Image der tschechischen Ratspräsidentschaft als guter Organisator zu erzeugen, war die Tatsache, dass die tschechische Ratspräsidentschaft auf die von Frankreich folgte, die von einer Reihe organisatorischer Probleme gekennzeichnet war. ${ }^{4}$

\section{Prioritäten der Ratspräsidentschaft: eine gemischte Bilanz}

Bei der Bewertung der Ziele der Ratspräsidentschaft müssen zwei Vorbehalte berücksichtigt werden: Erstens wurden diese Ziele, so sorgfältig sie auch geplant gewesen sein mögen, oft von unerwarteten Entwicklungen außerhalb der Europäischen Union (wie zum Beispiel der Gaskrise im Januar), innerhalb der Europäischen Union (wie zum Beispiel der Notwendigkeit, mit den ,Zugeständnissen“ an Irland umzugehen) sowie im Land, das die Ratspräsidentschaft hielt, selbst (wie zum Beispiel dem Sturz der Regierung) beeinflusst. Zweitens gab es zwei sehr unterschiedliche Prioritätenlisten - die drei Schlüsselziele: „Ökonomie, Energie und Europa in der Welt" und das wesentlich detailliertere, aber ebenso ehrgeizige Arbeitsprogramm der Ratspräsidentschaft - eine Unterscheidung, die bisher in nahezu allen Präsidentschaftsbewertungen unterblieben ist. ${ }^{5}$ Der Unterschied zwischen diesen beiden Ansätzen entspricht der Aufteilung der Arbeit zwischen der politischen Elite (die drei Schlüsselziele) und den Diplomaten und Beamten der mittleren Ebene und erklärt auch das oft erwähnte Schlagwort, das die tschechische Ratspräsidentschaft als ,gute Diplomatie, aber schlechte Politik' beschreibt. Tatsächlich war die Ratspräsidentschaft viel erfolgreicher in der Abarbeitung des Arbeitsprogramms als bei den Hauptzielen, deren Resultate eher gemischt ausfielen. ${ }^{6}$

Wenngleich sich diese Prioritäten auf die sechs Monate der tschechischen Ratspräsidentschaft beziehen, so sollten sie doch auch innerhalb des Präsidentschaftstrios (Frankreich, Tschechische Republik, Schweden) koordiniert werden. Doch dort wurde die Zusammenarbeit - wie auch in einigen anderen Bereichen - von einer eher gleichgültigen Haltung der französischen Diplomatie beeinträchtigt. Anders als Stockholm stützte sich Paris stark auf die einseitige Festlegung der eigenen Prioritäten während der französischen Ratspräsidentschaft und die geringe französische Bereitschaft zur Kooperation mit den beiden kleineren Staaten blieb ein Dauerthema während der gesamten tschechischen Ratspräsidentschaft.

3 Siehe Tschechische Ratspräsidentschaft: Summary of the Work of the Central Coordination Team during the Czech Presidency of the Council of the EU. Organisation, Logistics, and Communication, abrufbar unter: http:// www.eu2009.cz/scripts/file.php?id=61193\&down=yes (letzter Zugriff: 22.09.2009).

4 Zum Beispiel International Press Association: French Presidency Sub-standard, abrufbar unter: http:// www.api-ipa.eu/index.php?option=com_content\&task=view\&id=97\&Itemid=35 (letzter Zugriff: 22.09.2009). Für eine ähnliche Beurteilung siehe Aleksander Fuksiewicz/Agnieszka Łada: The Czech Presidency in the Council of the European Union - the view from Poland, Institute of Public Affairs research report, Warschau 2009, S. 25-26.

5 Für die drei Hauptziele siehe z.B. Mirek Topolánek: Message from Mirek Topolánek, Prime Minister of the Czech Republic, from 1 December 2008, abrufbar unter: http://www.eu2009.cz/en/czech-presidency/czechpresidency-4014/ (letzter Zugriff: 22.09.2009); für das Arbeitsprogramm siehe Tschechische Ratspräsidentschaft: Work Programme and Priorities of the Czech EU Presidency, abrufbar unter: http://www.eu2009.cz/en/ czech-presidency/programme-and-priorities/programme-and-priorities-479/ (letzter Zugriff: 22.09.2009).

6 Tschechische Ratspräsidentschaft: Achievements of the Czech Presidency. Europe without Barriers, abrufbar unter: http://www.eu2009.cz/scripts/file.php?id=60442\&down=yes (letzter Zugriff: 22.09.2009). 
Eine mögliche Erklärung - neben Präsident Nicolas Sarkozys Aktionismus - liegt in der substanziellen Überschneidung von Interessen zwischen der Tschechischen Republik und Schweden einerseits und einigen Unterschieden zwischen diesen beiden und Frankreich andererseits. Diese Unterschiede beinhalten eine breite Palette von Themen einschließlich der Erweiterung, der Östlichen Partnerschaft, der Gemeinsamen Agrarpolitik und einer Reihe institutioneller Fragen.

Die , nationale ' Festlegung der Prioritäten hatte bedauerliche Folgen, vor allem wenn von den drei Schlüsselzielen die Rede ist. Das zugrunde liegende Problem war, dass diese Prioritäten von Anfang an von der grundsätzlichen Überzeugung der seinerzeitigen Regierung und vor allem der ODS - gefärbt waren, dass das erste Ziel der Ratspräsidentschaft die Förderung , nationaler Interessen " und/oder , nationaler Interpretationen der Interessen der Europäischen Union' sein sollte und erst danach von dem klassischen Verständnis des Präsidentschaftslandes als Brückenbauer oder Vermittler die Rede sein sollte.

Getreu diesem Ansatz wurde die Europäische Union in Wirtschaftsfragen, neoliberalen Vorstellungen folgend, als ein freier Markt aufgefasst, in dem staatliche Interventionen abgelehnt, Subventionen reduziert und Entscheidungen in Brüssel so weit wie möglich vermieden werden sollten, vor allem, wenn sie in wirtschaftlich sensible Bereiche wie Steuern oder soziale Sicherheit überzugreifen drohten. Dieses Verständnis wäre nicht notwendigerweise von Nachteil gewesen, wenn es nicht die Finanzkrise gegeben hätte, die die Meinung sowohl in Europa wie in den USA veränderte und zu einer enormen Vorliebe für staatliche Interventionen und Regulierungen führte. Einige tschechische Politiker waren sich dieser Veränderung sehr wohl bewusst und bekämpften sie dennoch, wie zum Beispiel Staatspräsident Klaus, der sowohl die Krise als auch die Versuche, ihr zu entkommen, bei jeder sich bietenden Gelegenheit verharmloste. ${ }^{7}$ Andere wiederum realisierten vermutlich kaum, wie tief greifend diese Veränderung die politische Landschaft beeinflusste, und sagten ihre Meinung vielleicht zu offen - wie Premierminister Mirek Topolánek mit seiner berühmt-berüchtigten Aussage, in der er die neue Wirtschaftspolitik der USA als „Weg in die Hölle“ bezeichnete. ${ }^{8}$

Tatsächlich war es also kein Versehen oder Gleichgültigkeit, dass die tschechische Ratspräsidentschaft versuchte, die Anzahl und das Ausmaß von EU-weiten Krisenbekämpfungsmaßnahmen auf ein Minimum zu reduzieren. Stattdessen betonten tschechische Politiker, in diesem Fall mit ihren deutschen Amtskollegen, mit voller Absicht die Notwendigkeit nationaler Maßnahmen.

Da die Krise das Land später und schwächer traf als die meisten anderen Länder in der Region, wurde außerdem befürchtet, dass eine gemeinsame Aktion den Eindruck weiter verstärken würde, dass der Zustand des eigenen Landes so schlecht wie der von Ungarn oder der baltischen Länder wäre. Es kann daher nicht überraschen, dass die Bewertung der Ratspräsidentschaft durch den Vizepremierminister für Europäische Angelegenheiten von einem „koordinierten Ansatz“ der Mitgliedsländer" und nicht von einem ,gemeinsamen Ansatz“ sprach. Aus dem gleichen Grund führt der Evaluationsbericht unter den größten Erfolgen im Wirtschaftsbereich „die Zurückweisung protektionistischer Maßnahmen“ auf. ${ }^{10}$ Selbst als die Ratspräsidentschaft Schritte (zum Beispiel die Tagung des Europäischen Rates im März und das Europäische Konjunkturprogramm) unternommen hatte, schien die tschechische Diplomatie hierzu gezwungen worden zu sein, aus Furcht, sie könnte von dem hyperaktiven

7 Czech News Agency: EU using any opportunity to limit members' powers - Klaus, 24.07.2009.

8 Czech News Agency: USA's way out of crisis is road to hell - Topolanek, 25.03.2009.

9 Tschechische Ratspräsidentschaft: Achievements of the Czech Presidency, 2009, S. iv.

10 Ebenda, S. v. 
französischen Präsidenten in den Hintergrund gedrängt werden. Zudem war von Beginn an klar, dass die Europäische Kommission die zentrale treibende Kraft für diese Maßnahmen war und nicht die Ratspräsidentschaft.

Bezüglich der zweiten übergreifenden Priorität - Energie - war die tschechische Diplomatie sehr wohl für ,einen einheitlichen Ansatz“. ${ }^{11}$ Es ist unmöglich, die tschechischen Erfolge und Misserfolge in diesem Bereich zu bewerten ohne zu betonen, dass in den Köpfen tschechischer Politiker und Diplomaten dieses vorrangige Thema nahezu ausschließlich aus der externen EU-Energiesicherheit und dem internen EU-Energiemarkt bestand. Mit anderen Worten: Themen wie Klimawandel (Vorbereitung für den Gipfel in Kopenhagen im Dezember 2009) und erneuerbare Energieressourcen wurden mehr oder weniger an den Rand geschoben. Obwohl die Grünen, die während der Ratspräsidentschaft zur tschechischen Regierungskoalition gehörten, versuchten, diese Themen auf die Agenda der Ratspräsidentschaft zu setzen, hatten sie kaum Erfolg. Das soll nicht heißen, dass die Ratspräsidentschaft nicht die meisten ihrer Aufgaben im Bereich des Umweltschutzes, wie im Arbeitsprogramm vorgesehen, erfüllt hätte. Angesichts der Anzahl der Richtlinien war die Ratspräsidentschaft sogar ziemlich erfolgreich. Doch der Umweltschutz ist ein typisches Beispiel für einen Bereich, in dem Erfolg erreicht wurde trotz fehlender - und nicht dank - politischer Unterstützung der Agenda. Dies gilt vor allem für die Vorbereitungen des Gipfels von Kopenhagen zum Klimawandel, der von einigen hochrangigen tschechischen Politikern schlicht ignoriert wurde.

Die wachsenden Ängste gegenüber einem wieder erstarkenden Russland gekoppelt mit einer relativ großen Abhängigkeit von russischem Öl und Gas waren der Hauptgrund für die präzedenzlose Fokussierung auf Fragen der Energiesicherheit. Zugleich war ein Umweltbewusstsein noch nie tief verwurzelt in der ODS und die Lieblingsbeschäftigung des Staatspräsidenten, dessen Schatten noch immer über der Partei liegt, ist seit jeher der Kampf gegen Umweltschützer. ${ }^{12}$

Schwerpunkt in Sachen Energie, worauf sich die tschechische Ratspräsidentschaft stark konzentrierte, war die Wiederbelebung der Nabucco-Pipeline. Premierminister Topolánek erklärte dieses Projekt zu ,einem Test für die europäische Integration“ und zu einer Möglichkeit, „Freiheit für Europa“ zu sichern. ${ }^{13}$ Doch der Erfolg ist ein zweifelhafter: Einerseits hat der Prager Energiegipfel vom Mai geholfen, die bisher zögerliche Türkei davon zu überzeugen, sich diesem Projekt anzuschließen und weitere politische Unterstützung für die Pipeline seitens anderer möglicher Transitländer zu sichern. Andererseits fehlt immer noch die Garantie für ausreichende Gaslieferungen und der russische Widerstand gegen dieses Projekt ist sicherlich nicht geringer geworden.

Die dritte Priorität - Europa in der Welt - trägt ebenfalls den Stempel regionaler tschechischer Interessen, die im Osten und Südosten Europas liegen. Weil der tschechische Beitrittsfavorit auf dem Westlichen Balkan - Kroatien - seine territorialen Konflikte mit Slowenien nicht beenden konnte und der Beitrittsprozess gestoppt werden musste, hat sich die tschechische Aufmerksamkeit weiter ostwärts gewandt. Die Östliche Partnerschaft wurde das auffälligste Ergebnis der tschechischen Ratspräsidentschaft in diesem Bereich. Die Neubelebung der schwächelnden Europäischen Nachbarschaftspolitik, das Hauptanliegen des ursprüng-

11 Ebenda, S. vi.

12 Siehe z.B. Václav Klaus: Challenges of the Current Era, Rede in Washington D.C. im CATO Institute, 09.03.2007, abrufbar unter: http://www.klaus.cz/klaus2/asp/clanek.asp?id=3eLwSP6fD2kj (letzter Zugriff: 22.09.2009).

13 iDNES.cz: Plynovod Nabucco zajistí svobodu, ř́ká Topolánek (Gas-Pipeline Nabucco sichert Freiheit, sagt Topolánek), 28.01.2009. 
lich polnisch-schwedischen Vorschlags, sollte den Beziehungen der Europäischen Union zu den osteuropäischen Partnern neues Leben einhauchen. Ungeachtet des Prager Gipfels, der das Projekt lancierte, und der Tatsache, dass die Ratspräsidentschaft erfolgreich zusätzliche Finanzmittel für die Partnerschaft sichern konnte, bleibt ihr umfassender Langzeiterfolg an mehrere Bedingungen geknüpft. Vor allem konnte die tschechische Ratspräsidentschaft einige ihrer EU-Partner nicht von der Bedeutung des Gipfels für die gesamte Union überzeugen. Schlüsselfiguren, die die südliche Dimension der Europäischen Union repräsentieren, einschließlich der Ministerpräsidenten Silvio Berlusconi und José Luis Rodríguez Zapatero sowie Präsident Sarkozy, nahmen nicht an diesem Gipfel teil, ein deutliches Zeichen für ihr Desinteresse an diesem Projekt. Die Bedeutung der Östlichen Partnerschaft hängt auch ab von der Fähigkeit ihrer Unterstützer, deren kontinuierliches Arbeiten zu sichern, einschließlich ausreichender finanzieller Mittel und regelmäßiger EU-finanzierter Gipfeltreffen der teilnehmenden Regierungen.

Die tschechische Ratspräsidentschaft betonte energisch die Idee der Europäischen Union als eines ,global players', doch sie sah die Rolle der Europäischen Union wieder durch das Prisma tschechischer Interessen, wie die Regierung sie verstand, indem sie sich für eine einheitliche und selbstbewusste Politik gegenüber Russland einsetzte und für enge transatlantische Beziehungen auf der Grundlage der Überzeugung, dass die USA der wichtigste natürliche Partner und strategische Verbündete (und nicht ein Konkurrent) der Europäischen Union sind. Aus diesem Grund richtete sich die Energiepolitik der tschechischen Ratspräsidentschaft nicht nur auf die Absicherung der Stabilität von Energielieferungen als solcher, sondern auch auf eine Garantie der geopolitischen Unabhängigkeit der Europäischen Union (gegenüber Russland) und der Wettbewerbsfähigkeit der Europäischen Union auf globaler Ebene.

Hinsichtlich der Fähigkeit der tschechischen Diplomatie, auf unerwartete Entwicklungen in der Welt zu reagieren, war der Eindruck ebenfalls gemischt. Um nur zwei Beispiele zu geben, ein positives und ein negatives, sei auf die beiden Krisen verwiesen, denen sich die Ratspräsidentschaft gleich zu Beginn gegenüber sah. Im russisch-ukrainischen Gasstreit im Januar 2009 war die Pendeldiplomatie von Topolánek ein eher unerwarteter und doch überraschend effektiver Weg, den Konflikt zu lösen. Der Erfolg der Verhandlungen überraschte die EU-Offiziellen vermutlich ebenfalls - es sei daran erinnert, dass der Präsident der Europäischen Kommission, José Manuel Barroso, das Angebot der Ratspräsidentschaft verworfen hatte, sich an den Verhandlungen zu beteiligen. Andererseits zeigten die unpassenden Kommentare zu den jüngsten Gewaltausbrüchen im israelisch-palästinensischen Konflikt deutlich die Grenzen sowohl des tschechischen diplomatischen Geschicks als auch des geografischen Raumes, in dem das Land sich einbringen kann.

Insgesamt kann die Umsetzung der Prioritäten der Ratspräsidentschaft in vier Punkten zusammengefasst werden:

1. Sehr gute Resultate wurden erzielt bezüglich des Arbeitsprogramms der Ratspräsidentschaft, doch die Bilanz für die drei Hauptziele ist eher uneinheitlich.

2. Die drei Hauptziele wurden von der Regierung, von oben' bestimmt und waren daher stark politisch gefärbt: Als Folge davon wurde der Kampf gegen die Wirtschaftskrise zeitweise zu einem Kampf gegen Überregulierung, die Bedeutung von Energiefragen wurde überwiegend auf die Sicherung der Energieunabhängigkeit von Russland eingeschränkt und die außenpolitischen Aktivitäten der Ratspräsidentschaft zielten fast ausschließlich auf Osteuropa und die USA. 
3. Die Liste der sichtbarsten Erfolge beinhaltet die Wiederbelebung der Nabucco-Pipeline und die Lancierung der Östlichen Partnerschaft. Doch beide Projekte haben ihre besonderen Bedingungen und beide hängen vor allem von der Fähigkeit der Europäischen Union ab, sie auch nach dem Ende der tschechischen Ratspräsidentschaft hoch auf ihrer Agenda zu halten.

4. Zu den an den Rand gedrängten Prioritäten gehörten der Vertrag von Lissabon (geschuldet der komplizierten innenpolitischen Lage in einigen Mitgliedstaaten), Umweltprobleme (einschließlich Klimawandel und alternative Energiequellen), die Entwicklungshilfe und nicht zuletzt ein wirklich gemeinsamer Kampf gegen die Wirtschaftskrise.

\section{Außenwahrnehmungen: bestätigte Stereotypen}

Während die Verwirklichung von Prioritäten einen notwendigen Teil der Aktivitäten der Ratspräsidentschaft darstellt, obliegt die abschließende Bewertung hierüber zu einem großen Teil der Wahrnehmung durch Schlüsselinstitutionen der Europäischen Union (wie der Europäischen Kommission und dem Europäischen Parlament), durch renommierte Tageszeitungen und durch die bekanntesten Think-Tanks. Interessanterweise erfuhr die tschechische Ratspräsidentschaft ein großes Medienecho, sicherlich mehr als die erste Ratspräsidentschaft eines anderen neuen Mitgliedslandes (Slowenien). Die Frage ist jedoch, ob dieses Echo nicht eher den Fehlern und Fehltritten der Ratspräsidentschaft (wie der provokanten Kunstinstallation in Brüssel) geschuldet war. Der Schlüssel zum Verständnis der überwiegend negativen Sicht auf die tschechische Ratspräsidentschaft liegt dabei in der Grundauffassung, dass es dem tschechischen EU-Vorsitz trotz des erwähnten Tatendrangs und der Ambitionen nicht gelungen ist, das Etikett als Euroskeptiker loszuwerden. Mehr noch, das Image der ,euroskeptischen Tschechen“ wurde von Václav Klaus' fortgesetzten Attacken auf den Vertrag von Lissabon, auf die Bedeutung der Institution der EU-Ratspräsidentschaft und ganz allgemein auf die Legitimität der Europäischen Union weiter gepflegt, was in der europäischen Presse intensiv kommentiert wurde. ${ }^{14}$

Die eifrige Vorbereitungsphase, die PR-Kampagne und die Person von Premierminister Topolánek und anderen tschechischen Politikern suggerierten, dass die Ratspräsidentschaft sich eher um die Förderung von ,Visionen und Agenden ' drehte als darum, die Rolle eines unparteiischen und ehrlichen Vermittlers, eines Brückenbauers oder unauffälligen Managers zu übernehmen. Daher überrascht es nicht, dass die Ratspräsidentschaft unterschiedlich bewertet wurde. ${ }^{15}$ Länder und Akteure, die die tschechische Vision und Agenda teilen, beurteilten die Ratspräsidentschaft gemeinhin positiv. Die Ratspräsidentschaft erhielt gute Noten (,nicht so schlecht, wie es schien') von den neuen Mitgliedstaaten, die in den Fragen der geopolitischen Orientierung der Ratspräsidentschaft gen Osteuropa, der Fokussierung auf Energiefragen (externe Energiesicherheit) und der Verteidigung des freien Binnenmarktes gleicher Meinung sind. ${ }^{16}$ Auch die Kommission teilte ihre Zufriedenheit mit, besonders wegen der tschechischen Offensive in der Energiepolitik. Think-Tanks in Brüssel konzentrierten sich überwiegend auf die inhaltlichen Errungenschaften der Ratspräsidentschaft, auch mit unvoreingenommenen Urteilen. ${ }^{17}$

14 Maurin Ricard: Vaclav Klaus, trouble-fête de l'Europe, in: Le Figaro, 01.01.2009; Ian Traynor: Fears as Czech Republic takes over helm of EU, in: The Guardian, 01.01.2009.

15 Kathleen Moore: ,Chaotic' Czech EU Presidency Comes To Close, in: Radio Free Europe/Radio Liberty, 30.06.2009. Die tschechische Ratspräsidentschaft wurde in der französischen Presse besonders negativ beurteilt. Siehe Stéphane Kovacs: UE: la Suède se prépare à une présidence tourmentée, in: Le Figaro, 23.06.2009.

16 Fuksiewicz/Łada: The Czech Presidency in the Council, 2009.

17 Piotr Maciej Kaczynski: How the Czech Presidency Performed up to Now, in: European Voice, 26.03.2009. 
Langjährige Mitgliedstaaten, die immer wieder über das Funktionieren der erweiterten Europäischen Union besorgt sind, legten ihr Augenmerk eher auf die prozeduralen Aspekte der Ratspräsidentschaft (darauf, wie die Tschechische Republik diese Rolle meisterte) als auf die inhaltlich-politischen Ziele und deren Verwirklichung. Ein Ergebnis dieser Haltung war, dass Befürchtungen vor der Ratspräsidentschaft oft auf (mangelnde) tschechische organisatorische, leitende und diplomatische Fähigkeiten, fehlenden Enthusiasmus und zu wenig Verantwortung abhoben. Nach anfänglichem kurzem Schwanken erwies sich die tschechische Diplomatie jedoch als ziemlich aktiv und widerlegte den Vorwurf mangelnder Begeisterung. Die Pendeldiplomatie zwischen Kiew und Moskau während der Gaskrise erhielt (von Überraschung geprägtes) Lob und zeigte, dass auch ein neues (und kleines) Mitgliedsland über Fähigkeiten und Kapazitäten verfügt, die in Verhandlungen über eine schwere außenpolitische Krise vonnöten sind.

Doch tschechischer Aktivismus und Mut hatten auch ihre Schattenseiten. Die hastige proisraelische Stellungnahme während der Gaza-Krise und ein unpassender Kommentar über Barack Obamas Pläne zur Ankurbelung der Wirtschaft - ein „Weg in die Hölle“ - wurden als undiplomatisch und unprofessionell empfunden. Derartige Ausrutscher ließen Fragen laut werden wie es um die Fähigkeit in Tschechien stand, zu verstehen, ,welches die Aufgabe der EU-Präsidentschaft sei“ “. ${ }^{18}$ Einige Insider kritisierten auch die Strategie, neue Initiativen „mit dem Fallschirm“ von Prag aus zu lancieren, ohne sich vorher mit europäischen Kollegen abzusprechen. ${ }^{19}$ Hinzu kam, dass Mut und Unternehmungsgeist sich manchmal in Arroganz und Moralpredigten wandelten. So wurden zum Beispiel sogar die, die dem Inhalt der Argumente von Staatspräsident Klaus ${ }^{20}$ über die unwillkommene Erweiterung der Zuständigkeiten der Europäischen Union wohlwollend gegenüberstanden, von seinem „,bizarren Schwadronieren“ über die Ähnlichkeiten zwischen der Europäischen Union und der Sowjetunion abgeschreckt. ${ }^{21}$

Insgesamt stand die Tschechische Republik unter genauer Beobachtung und es passt in diesen Kontext, dass das Misstrauensvotum vom 24. März 2009 weit verbreitete Kritik, vor allem seitens der langjährigen Mitgliedsländer und des Europäischen Parlaments, nach sich zog. Der Sturz der tschechischen Regierung wurde als Akt der Verantwortungslosigkeit und Unreife bezeichnet, der nicht nur das Image der Tschechischen Republik, sondern auch der Europäischen Union als solcher beschädigt hätte. Obwohl die Übergangsregierung die tägliche technische Agenda gut bewältigte, gewann das Argument, dass die tschechische innenpolitische Auseinandersetzung die Europäische Union ohne politische Führung ließ, immer mehr an Boden. ${ }^{22}$ Die harsche Beurteilung der politischen Repräsentanten stand allerdings oft im Gegensatz zu einer Wertschätzung tschechischer Diplomaten und Funktionäre. ${ }^{23}$

18 DW-World.de: Czech Leader's 'Hell' Remark Fans Flames of EU-US Dissent, 26.03.2009.

19 Andrew Rettman: Czech presidency limps off EU stage, in: EUobserver.com, 30.06.2009.

20 Viele Journalisten, Kommentatoren und selbst Politiker erlebten und bewerteten die Ratspräsidentschaft nahezu ausschließlich durch Staatspräsident Klaus, dabei (fälschlicherweise) annehmend, dass er derjenige sei, der die Verantwortung für die Ratspräsidentschaft habe.

21 James Joyner: EU President Vaclav Klaus Condemns EU, in: New Atlanticist. Policy and Analysis Blog, 20.02.2009. Siehe auch The Economist: Grumpy Uncle Vaclav, 04.12.2008.

22 Eric Bonse/Stefan Menzel: EU verliert politische Führung, in: Handelsblatt, 06.04.2009.

23 Tony Barber: Switch-off ends Czech's life support presidency, in: Financial Times, 16.06.2009. Siehe auch EarthTimes: Some wins, but Czech EU presidency seen as chaotic failure - Feature, 28.06.2009. 
Insgesamt lässt sich die Wahrnehmung der Ratspräsidentschaft wie folgt zusammenfassen:

1. Die Ratspräsidentschaft begann mit dem Ehrgeiz, ,Spuren hinterlassen ' zu wollen und das Image der Tschechischen Republik zu verbessern und versuchte dabei, für die tschechische Vision der Europäischen Union zu werben.

2. Das Land war jedoch nicht in der Lage, das stereotype Image eines tiefen Euroskeptizismus, verkörpert vom tschechischen Staatspräsidenten Václav Klaus, abzustreifen.

3. Nach dem Sturz der Regierung gaben auch die treuesten Befürworter der Ratspräsidentschaft zu, dass die innenpolitische Situation einen außerordentlichen Fehler darstellte, der den Ruf des Landes im Ausland stark beschädigte.

4. Die Gesamtbewertung der Ratspräsidentschaft ist eher gemischt, mit mehr positiven Meinungen, die aus osteuropäischen oder wirtschaftlich liberaleren Mitgliedstaaten kommen. Die Hauptakteure in Brüssel, mit der Europäischen Kommission und wichtigen ThinkTanks, sprachen von einem gemischten Ergebnis. Andere, vor allem Frankreich aus dem Kreis der Mitgliedstaaten und das Europäische Parlament aus dem Kreis der EU-Institutionen, waren viel kritischer.

\section{Die innenpolitischen Auswirkungen: die Marginalisierung der Euroskeptiker}

Mit wenigen Ausnahmen wie der Östlichen Partnerschaft oder der Nabucco-Pipeline waren die Ergebnisse der tschechischen Ratspräsidentschaft für die Europäische Union als Ganzes eher unbedeutend. Die innenpolitischen Auswirkungen der Ratspräsidentschaft waren jedoch enorm. Das wichtigste Ergebnis ist der Wechsel der ODS, der einstmals stärksten euroskeptischen Partei des Landes, hin zum EU-Mainstream. Die Ratspräsidentschaft machte Premierminister Topolánek, der früher den Vertrag von Lissabon mit starken Worten abgelehnt hatte, zu einem seiner innenpolitischen Hauptbefürworter. Die tschechischen Euroskeptiker in der ODS, die traditionell jeglicher Ausweitung der EU-Zuständigkeiten misstrauten, wandelten sich über Nacht in pragmatische Förderer einer gemeinsamen Energiepolitik. ${ }^{24}$ Die ODS betrachtete traditionell auf ähnlich kritische Weise die Gemeinsame Außen- und Sicherheitspolitik und Europäische Sicherheits- und Verteidigungspolitik der Europäischen Union. Doch in den letzten Jahren gab es Anzeichen für einen Wandel in der Sprache, die implizit oder explizit eine umfassendere und von Prinzipien getragene Außenpolitik der Europäischen Union befürwortete und die die Idee der Europäischen Union als einem globalen politischen Akteur guthieß.25 Dieser Trend gipfelte während der Ratspräsidentschaft darin, dass Premierminister Topolánek pragmatisch einer einheitlichen, kohärenten und unabhängigen Position der Europäischen Union gegenüber Russland und den östlichen Nachbarn der Europäischen Union in so unterschiedlichen Bereichen wie der politischen Zusammenarbeit, wirtschaftlichen Bindungen und der Energiesicherheit das Wort redete. Ihm folgend realisierte ein großer Teil seiner Partei, dass die ideologischen Vorbehalte gegenüber der europäischen Integration sie eher zu Außenseitern machen, sowohl im Umgang mit ihren EU-Partnern als auch gegenüber ihren eigenen Wählern, die üb-

24 Mirek Topolánek: Speech by Mirek Topolánek at the Nabucco summit, 11.02.2009, abrufbar unter: http:// www.eu2009.cz/en/news-and-documents/speeches-interviews/speech-by-mirek-topolanek-at-nabucco-summit-7778/ (letzter Zugriff: 22.09.2009).

25 Vít Beneš: The Czech discourse on EU's external activities, in: Graham Avery/Anne Faber/Anne Schmidt (Hrsg.): Enlarging the European Union: Effects on the new member states and the EU, Trans European Policy Studies Association, Brüssel 2009, S. 47-50. Zur Illustration siehe Mirek Topolánek: Na jednání o smlouvě EU budeme psi obranáři, in: Euro, 01.10.2007. 
licherweise stark pro-europäisch waren. Die ODS-Führung konnte diese Diskrepanz zwischen Parteirhetorik und den Ansichten ihrer Wähler solange ignorieren, wie die Europäische Union nicht das Hauptthema war. Aber mit dem Beginn der Ratspräsidentschaft trat EU-bezogene Politik in den Vordergrund und die ODS musste sich diesem doppelten Druck beugen.

Eine zweite Konsequenz war die zunehmende Isolierung von Staatspräsident Klaus. Vom Vertrag von Lissabon bis hin zu Klimawandel und Wirtschaft beherzigte die ODS nicht länger seine Ratschläge aus dem Elfenbeinturm. Während Präsident Klaus in früheren Jahren ziemlich erfolgreich mit Konspirationstheorien, wonach die Europäische Union unter dem Einfluss sozialistisch gesinnter europäischer Bürokraten stand, agierte, reduzierte die direkte Erfahrung der ODS mit dem EU-Geschäft die Plausibilität der präsidialen Argumentation. Die Ratspräsidentschaft ermöglichte es den tschechischen Euroskeptikern, die Europäische Union in einem anderen Licht zu sehen: eine Organisation, gemanagt von Diplomaten aus 27 Mitgliedstaaten, wo die ,Bürokraten“ der Kommission oft die Sache von Liberalismus und EU-Erweiterung verteidigen. Die Entfernung zwischen der ODS und Präsident Klaus wurde während der Ratspräsidentschaft stetig größer und im April 2009 konnte man sogar ranghohe Politiker der ODS erleben, wie sie über die Involvierung des Staatspräsidenten in den Sturz der Regierung spekulierten. Daraus resultierte, dass Staatspräsident Klaus, der immer als gewiefter Machiavellist eingeschätzt wurde, an der innenpolitischen Front klar geschlagen wurde. Beim Austritt aus der Partei (im Herbst 2008), die er selbst gegründet hatte, verhehlte er nicht seine Enttäuschung und übertrug seine Unterstützung auf die neu gegründeten euroskeptischen Parteien, die bei den Wahlen zum Europäischen Parlament jedoch deutlich geschlagen wurden. Trotz (oder wegen) seiner innenpolitischen Demütigung will der bedrängte Staatspräsident gemeinsam mit einer Handvoll loyaler Senatoren seinen Kampf gegen den Vertrag von Lissabon fortsetzen, eine Aufgabe, die durch die anfänglich halbherzige Unterstützung der tschechischen Ratspräsidentschaft für die Ratifizierung des Vertrages erleichtert wurde. ${ }^{26}$

Die dritte Auswirkung auf die politische Lage in Tschechien betrifft die Verschiebung der Links-Rechts-Achse im politischen Spektrum bezüglich der europäischen Integration. Da die stärkste linke Kraft des Landes, die sozialdemokratische Partei (ČSSD) eine Hauptrolle beim Sturz der Regierung spielte, wurde deutlich, dass die Partei einen innenpolitischen Sieg über einen störungsfreien Ablauf der EU-Ratspräsidentschaft stellte. Addiert man dies zu den wachsenden Zweifeln der ČSSD am vermeintlichen Liberalismus der Europäischen Union und ihrer Unwilligkeit, das Haushaltsdefizit auf einem Niveau zu halten, das einen schnellen Beitritt zur gemeinsamen Währung erlauben würde, scheint es, als würde die Aufteilung in eine pro-europäische Linke und euroskeptische Rechte bald obsolet werden. Stattdessen wird das Land vermutlich zwei große pro-europäische Parteien bekommen mit unterschiedlichen Vorstellungen darüber, wie die Europäische Union funktionieren soll.

\section{Fazit}

Die tschechische Ratspräsidentschaft begann ziemlich ehrgeizig, aber in einer Atmosphäre von niedrigen Erwartungen und Skepsis. Bedauerlicherweise trieb die tschechische politische Szene selbstbewusst ihre politische Botschaft auf die Spitze ohne zu bemerken, dass ihr provokanter Stil und ihr konfrontatives Vokabular die bereits existierenden Vorurteile und das Misstrauen nur bestätigten und bekräftigten. Das geringe Bewusstsein über die

26 Siehe Petr Kratochvíl: The Czech Republic: Klaus the last to sign..., in: Trans European Policy Studies Association: Lisbon Treaty Ratification Monitor -2nd issue, S. 2. 
Verknüpfung von Innenpolitik und internationaler Politik (gekrönt vom Sturz der Regierung) führte verständlicherweise zu überwiegend negativen Bewertungen für die tschechischen politischen Vertreter - vor allem unter den langjährigen Mitgliedstaaten, die über das interne Funktionieren der Europäischen Union und ihre globale Rolle besorgt waren. Vor diesem Hintergrund gibt es keinen Zweifel, dass die tschechische Ratspräsidentschaft den kleinen und/oder neuen Mitgliedstaaten ${ }^{27}$ einen Bärendienst erwiesen hat und die Argumentation für einen dauerhafteren Präsidenten des Europäischen Rates, wie im Vertrag von Lissabon vorgesehen, untermauerte.

Doch obwohl dieses Bild weitgehend korrekt ist, muss angemerkt werden, dass die tschechische Ratspräsidentschaft einige Ziele von gemeinsamem Interesse für die gesamte Europäische Union erreicht hat. Vor allem die erfolgreichen tschechischen Bemühungen, die Nachbarschaft der Europäischen Union zu stabilisieren, und Prags Aktivitäten auf dem Feld der Energiesicherheit haben das Potenzial, diese Ratspräsidentschaft zu überdauern. Auch sollte die innenpolitische Verschiebung hin zum Mainstream der Europäischen Union nicht unterbewertet werden. Die Tschechische Republik (zusammen mit Großbritannien) gehört zu den Ländern, in denen der Vertrag von Lissabon vor größten Problemen steht. Sollte allerdings die Europäisierung der Mehrheit der tschechischen Euroskeptiker zusammen mit der Marginalisierung der anderen Euroskeptiker ein dauerhaftes Phänomen sein, könnte sich die Haltung der tschechischen politischen Elite zu einer weiteren Vertiefung des Integrationsprozesses einschneidend verändern. Inwieweit die tschechischen Politiker die gelernten Lektionen beherzigen werden, wird spätestens in 14 Jahren deutlich werden, wenn das Land die Chance haben wird, ein weiteres Mal der Europäischen Union vorzusitzen.

Übersetzung aus dem Englischen von Gudrun Staedel-Schneider.

27 Fuksiewicz/Łada: The Czech Presidency in the Council, 2009. 